\title{
Impaired nonverbal working memory in high-functioning autism spectrum disorder
}

\author{
Atusa Rabiee ${ }^{1}$, Behnoosh Vasaghi-Gharamaleki*2, Sayyed Ali Samadi ${ }^{3}$, Younes Amiri-Shavaki ${ }^{1}$, \\ Javad Alaghband-rad ${ }^{4}$, Saba Seyedin ${ }^{5}$, Soode Hosseini ${ }^{6}$
}

\section{Abstract}

Background: Past studies have documented working memory impairment in participants with high-functioning Autism Spectrum Disorders (IQ>70), but inconsistent findings have been reported. One possibility is the existence of verbal responses in the evaluation of working memory performance. The aim of the current study is to examine the working memory performance and its correlation with a prominent deficit in participants with high-functioning Autism Spectrum Disorders by non-verbal working memory tasks compared with typically developing samples.

Methods: The current study is a cross-sectional, comparative study. The working memory performance of the 30 participants with high-functioning Autism Spectrum Disorders (7-16 years) and 30 typically developing was compared by working memory subtests of the Leiter International Performance Scale-Revised in Tehran, Iran. Two groups were matched for age and gender. ANOVA, ANCOVA, repeated measures ANOVA and Pearson's correlation coefficient were used to analyze the data statistically. The significance value was set at $\mathrm{p}<0.05$.

Results: The results showed that if the effect of FSIQ (full-scale intelligence quotient) were controlled individuals with highfunctioning Autism Spectrum Disorders exhibited significant impairment in the Reverse Memory subtest ( $\mathrm{p}=0.001)$. Also, unpredictably Forward Memory $(r=0.38, p=0.03)$ and Reverse Memory tasks $(r=0.38, p=0.03)$ displayed a significant positive correlation with the Social interaction subscale of the Gilliam Autism Rating Scale (Second Edition).

Conclusion: It seems that nonverbal working memory is impaired in persons with high-functioning Autism Spectrum Disorders. Results of the current study revealed that factors like complexity and cognitive load of tasks may influence working memory performance in individuals with high-functioning Autism Spectrum Disorders.

Keywords: Autism spectrum disorder, Executive function, Working memory

Conflicts of Interest: None declared

Funding: Iran University of Medical Sciences, Tehran, Iran

\section{*This work has been published under CC BY-NC-SA 1.0 license.}

Copyright $\odot$ Iran University of Medical Sciences

Cite this article as: Rabiee A, Vasaghi-Gharamaleki B, Samadi SA, Amiri-Shavaki Y, Alaghband-rad J, Seyedin S, Hosseini S. Impaired nonverbal working memory in high-functioning autism spectrum disorder. Med J Islam Repub Iran. 2018 (3 Nov);32:107. https://doi.org/10.14196/mjiri.32.107

\section{Introduction}

Autism Spectrum Disorder (ASD), a heterogeneous set of neurodevelopmental disorders, is characterized by persistent deficits in social communication. Other characteris-

Corresponding author: Dr Behnoosh Vasaghi-Gharamaleki, vasaghi.b@iums.ac.ir

1. Department of Speech and Language Pathology, School of Rehabilitation Sciences, Iran University of Medical Sciences, Tehran, Iran

2. Department of Basic Sciences, School of Rehabilitation Sciences, Iran University of Medical Sciences, Tehran, Iran

3. Institute of Nursing and Health Research, University of Ulster, Northern Ireland, UK

4. Department of Psychiatry and Psychology Research Center, Roozbeh Hospital, Tehran University of Medical Sciences, Tehran, Iran

5. Department of Speech and Language Pathology, School of Rehabilitation Sciences, Tehran University of Medical Sciences, Tehran, Iran

6. Department of Psychology, School of Education and Psychology, Alzahra University, Tehran, Iran tics of ASD are the presence of stereotypic patterns of behaviors across several contexts with or without accompanying intellectual disability (1). High functioning ASD does not appear in the Diagnostic and Statistical Manual of

$\uparrow$ What is "already known" in this topic:

Some research was done in the developed country to evaluate working memory in individuals with high-functioning Autism Spectrum Disorders, but inconsistent findings have been reported. One possibility is the existence of verbal responses in the evaluation of working memory performance.

\section{$\rightarrow$ What this article adds:}

Working memory is impaired even if the effect of the verbal response were eliminated in the evaluation of working memory, but when the effect of full-scale intelligence quotient is controlled all the working memory tasks are not suitable to detect these problems. 
Mental Disorders (DSM) as a separate diagnosis but is a subtype of autism without any cognitive delay. The fullscale intelligence quotient (FSIQ) of them is reported above 70 (2). The prevalence of ASD is approximately 95 per 10,000 population in Iran (3). There is the significant malefemale ratio in the ASD and high-functioning ASD. The most widely reported is a $4: 1$ or $5: 1$ male: female ratio in the ASD prevalence (4). In high-functioning ASD the sex ratio is even higher. The ratio is reported from 5:1 to a maximum of 9:1 (5). The etiology of ASD has remained unknown (6). Many theories try to describe the prominent deficits of ASD. The Executive Dysfunction Theory is one of them. Executive function includes several abilities related to working memory (WM) as the main component (7). WM is defined as a process for the temporary holding and manipulating of information (8). WM is important in academic learning and daily living (9). The small number of studies investigated the relationship between prominent deficits of the ASD and WM impairment (7). In some of these studies, the authors reported a negative correlation between WM performance and the prominent deficits of ASD $(10,11)$. So, it is important to investigate WM impairments in individuals with ASD. There are a considerable amount of studies done in the developed countries on exploring the WM impairment of individuals with high-functioning ASD. There is inconsistent in the reported results of these studies. While the results of some research support an impaired WM (12), others have found evidence of relatively intact WM (13). Kercood et al. (2014) published a review of literature in WM and autism research and indicated that the arrangement of WM tasks might define whether or not functioning is impaired and the existence of verbal responses in the evaluation of WM might be a confounding variable and should be considered in future research (14).

To evaluate the cognitive functioning of people between 2 and 21 years old, the Leiter International Performance Scale-Revised (Leiter-R) was designed (15). This scale is a non-verbal and culturally free instrument. Because the Leiter- $\mathrm{R}$ is nonverbal, it is particularly appropriate for assessment of younger children with little or no language skills such as ASD. The Leiter is divided into two groups, (Visualization and Reasoning Battery (VR) and Attention and Memory Battery (AM)), and each group contains ten subtests. The VR and AM Battery may be administered separately or together, depending on the clinical necessity (16). Some studies explored the profile of VR Battery in children with ASD. These studies highly recommended using Leiter-R for assessment of WM in children with ASD (17) and showed a good competence in visuospatial disembedding and detail-focused processing and incompetence in abstraction and concept formation (18). To the authors' knowledge, no data exist on subtests of AM Battery in participants with ASD. However, some studies explored the subtests of AM Battery in fragile X syndrome (FXS) and found relative difficulty on WM tasks of the Leiter-R in children with FXS $(19,20)$.

Some studies have been performed on WM in persons with high functioning ASD in developed countries; however, their results are contradictory $(7,14)$. Previous studies showed the existence of verbal responses in the evaluation of WM might be a confounding variable and should be considered in future research (14). It seems that culture influences in working memory (21). Additionally, there is a dearth of similar data for developing countries for this topic, so the aim of the present study was to investigate WM performance in persons with high-functioning ASD and compare it to TD participants and to carefully look at patterns of relative strengths and weaknesses in each group, separately. Also, another aim of the present study is to research the possible correlation between prominent deficits of ASD that assessed through Gilliam Autism Rating ScaleSecond Edition (GARS-2) (22) and the WM tasks of LeiterR.

\section{Methods}

\section{Participants}

Participants with high-functioning ASD: In the present study, 30 children and adolescents with a confirmed diagnosis of high-functioning ASD aged 7 to 16 years (mean=133, SD=33 months) were recruited. The samples were selected using convenience sampling method. Participants in the current study were recruited from 121 people (7-16 years) with a diagnosis of high-functioning ASD. Special centers (the Iranian Special Education Organization, the Autism Charity, Roozbeh Psychiatric Hospital, the specialized clinics of schools of rehabilitation sciences, and ten specialized clinics for children with autism) referred these patients to the first author.

In order to confirm the diagnosis of high functioning ASD, GARS-2 and Wechsler Intelligence Scale for Children-Fourth Edition (WISC-IV)(23) were used. A trained speech and language pathologist (Ph.D. candidate) used GARS-2 and a well-trained and experienced psychologist (MSc degree) measured the intellectual levels of individuals. Participants, which were included in the study, were ones who obtained a standard score equal to or above 70 on the WISC-IV and a cut-off score on the GARS-2.

The subsequent criteria (24) were considered to exclude the participants from the study :

- Metabolic disorders and/or genetic syndromes which are known as comorbid conditions.

- A previous history of neurological diseases (eg. previous history of trauma, tumors, brain lesions, stroke or a medical problem such as epilepsy or Tourette syndrome).

- Further medical difficulties affecting cognition, comorbid mental disorders like schizophrenia or bipolar disorder, and special visual, hearing or motor problems which prevent performance in the study.

Conclusively, a total of 30 participants, including 27 boys and 3 girls, with high-functioning ASD were recruited in this research. The average age of participants (both genders) was 11.08 years. The socioeconomic status of the 7 families of these participants $(23 \%)$ was rated as 3 (to be in the middle class), 9 families (30\%) was rated upper than 3 (in the upper middle class) and 14 families (47\%) was rated lower than 3 (in the lower middle class). Assessments were carried out in a quiet room with appropriate light and temperature. 


\section{TD participants}

Twenty-seven boys and three girls (a total of 30 typically developing children) comprised the control group. They were matched based on gender and age (mean $=134, \mathrm{SD}=32$ months) with participants with high-functioning ASD. The TD participants control group recruited via cluster sampling from public schools in Tehran. Some of the municipality districts of Tehran were randomly selected, and an all-boys' and an all-girls' school were randomly selected from the primary and lower secondary schools of each district. No evidence of behavioral problems or a history of relevant diseases were detected according to the Strengths and Difficulties Questionnaire (SDQ), which will be described later in the Research Tools section and also a semi-structured interview. Situations disqualifying the participants were personal or family histories of psychiatric, neurological or other medical conditions affecting brain development. A typical academic background was existent in TD children in this sample. WISC-IV was used to estimate their intellectual levels of participants.

\section{Research tools}

The Wechsler Intelligence Scale-Fourth Edition (WISC$I V)$ : The Wechsler Intelligence Scale (WISC) is an intelligence test for youngsters ranging in ages 6 to 16 years old. The WISC-IV has four indices, ten core subtests, and five complementary subtests. The present study used the Persian version of the WISC-IV. The validity and reliability of this scale were evaluated on 872 Iranian people aged $6-16$ years. The test-retest reliability values were 0.91 for FSIQ and between 0.65 to 0.94 for its subtests (25).

WM subtests of The Leiter International Performance Scale-Revised (Leiter-R): Four subtests from the Attention and Memory Battery (AM) suitable for ages 6 to 20 years (Associated Pairs, Forward Memory, Reverse Memory and Spatial Memory) were used for more precise examination of WM ability. In Associated Pairs, pairs of pictured objects are revealed for a short time (5-10 seconds) then the participant is asked to put the pictured objects cards on a blank matrix in the right place. The ability to recall meaningful and non-meaningful associations is considered. For the Spatial Memory subtest, a demonstration of pictures in a matrix is shown for a short time of ten seconds. The participant has to place cards of the pictured objects on a blank matrix in the accurate positions. The assignment starts and ends with a single picture in a two-box matrix and eight pictures in a 12-box matrix, respectively. Credit on a given sample is received when the youngster puts all the pictures correctly on the matrix. The assignment is terminated after six errors one after the other. For the Forward Memory subtest, the examiner points to a picture every second, and then the youngster must show the equivalent picture with the same organization. The number of pictures escalates but begins with just one picture. The task terminates when six cumulative sequence errors have been made. The procedure in the Reverse Memory is analogous to the Forward Memory subtest; this means that the youngster shows the exact pictures in a reversed order.

The Persian version of the test was normalized by the Iranian Special Education Organization and psychometric properties of this scale were investigated in a national scale study.

The norms are used by Iranian Special Education organization with the purpose of diagnosis of children with special need annually in the national elementary school entry evaluation plan called "Sanjesh" (evaluation in Persian). The organization classified the statistical data and the test content as top confidential because of the possible misusage by nonofficial parties. No journal publication has been done yet about the so-called collected official information by this organization. The present study benefited assistance from the official test administrators and therefore was able to use the Iranian Special Education Organization norms.

The Gilliam Autism Rating Scale-Second Edition (GARS2): The GARS-2 is an instrument that assists clinicians in recognizing and diagnosing autism in individuals with the age range of 3 to 22 years. The scale has 42 items in three subscales (stereotyped behaviors, communication, and social interaction). The Persian version of the scale was used for this study. The psychometric properties of GARS-2 were evaluated in 658 children (442 with Autism; 112 intellectually disabled and 102 typically developing). The internal consistency of the subscales stereotyped behavior, Communication and Social interaction were reported to be $0.84,0.87$ and 0.88 respectively (26).

The Strengths and Difficulties Questionnaire (SDQ): The SDQ is a brief screening tool. Three to 16-year-olds take this test so we can determine emotional and behavioral difficulties in children and adolescents (27). The Persian version of this questionnaire test is a reliable and valid instrument $(28,29)$. The Validation of SDQ was performed on 600 children aged between 6 and 12 years old. The internal consistency was reported (0.73) and concurrent validity of this questionnaire was good.

The self-administered demographic and economic data gathering: Parental data is included in this form (their employment status, annual income, and level of education). It also includes questions about children's demographic information. In this research, the socioeconomic status of families was calculated based on the three variables (family income, parental education, and occupational status). According to the results of a study by Kazemipour (1999), each variable classified as a 5-point scale. The average of these three variables determined SES (30).

\section{Ethics approval}

This study was approved by the ethics committee of the Iran University of Medical Sciences (ir.iums.rec. 1394.9211363204). The parents of the participants signed a written approval form. Verbal approval was obtained from the participants.

\section{Statistical analysis}

The normality of the data was tested by the KolmogorovSmirnov test. The Analyses of Variance (ANOVA) and repeated measures ANOVA were used to examine the intragroup and intergroup comparisons on different WM subtests. Analyses of Covariance (ANCOVA) was used to control the effect of FSIQ on WM tasks. The Partial Eta Squared was used to evaluate the outcome of size on the 
ANOVA and ANCOVA. We examined the relationship between the GARS-2 with WM subtests of Leiter-R by Pearson's correlation coefficient. Analyses were done using SPSS 16 and a $p<0.05$ was the level of the statistical significance in all statistical tests.

\section{Results}

One-Sample Kolmogorov-Smirnov test in the WM subtests confirmed the normal distribution of the data in the WM subtests of the ASD group (Associated Pairs $(\mathrm{p}=0.96)$, Forward Memory ( $\mathrm{p}=0.59)$, Reverse Memory (0.84), Spatial Memory (0.27)) and TD group (Associated Pairs (0.07), Forward Memory (0.19), Reverse Memory (0.90), Spatial Memory (0.68)).

\section{Intergroup Comparisons}

Characteristics of two groups of participants including age, and FSIQ and the results of the GARS-2 in participants with ASD are shown in Table 1.

Age and gender were not significantly different in the two groups, but a significant difference was found in the FSIQ between the two groups. The matter of using FSIQ as a covariate in analyses of data has not been resolved yet in people with psychiatric disorders. Therefore the analyses were performed with and also without FSIQ as a covariate (31).

Analysis of each of the WM tasks between the high-functioning ASD and TD groups as shown in Figure 1, was revealed that participants with ASD were weaker than TD participants in all WM subtests (Associated Pairs ( $F_{(1,}$ $\left.{ }_{58}\right)=10.56, \mathrm{p}=0.002$, Partial $\left.\mathrm{\eta}^{2}=0.15\right)$, Forward Memory $(\mathrm{F}$ $(1,58)=17.12, \mathrm{P}=0.001$, Partial $\left.\mathrm{\eta}^{2}=0.22\right)$, Reverse Memory $\left(F_{(1,58)}=54.96, p=0.001\right.$, Partial $\left.\mathrm{j}^{2}=0.48\right)$ and Spatial Memory $\left(F_{(1,58)}=22.60, p=0.001\right.$, Partial $\left.\left.\mathrm{p}^{2}=0.28\right)\right)$.

After covarying the FSIQ and evaluating other variables as dependent variables, it was discovered that participants with high-functioning ASD were significantly punier than the TD control group in the Reverse Memory $\left(\mathrm{F}_{(1,57)}=5.93\right.$, $\mathrm{p}=0.01$, Partial $\mathrm{y}^{2}=0.09$ ).

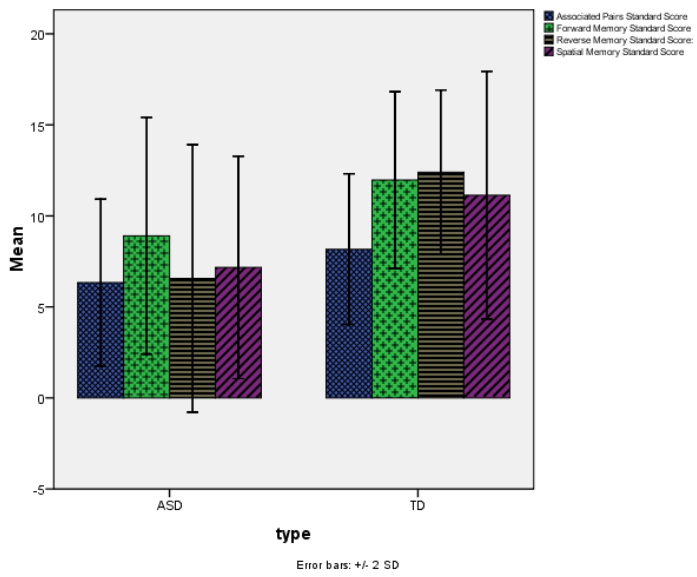

Fig. 1. Bar-chart of the working memory tasks of Leiter-R by type (the high-functioning Autism Spectrum Disorders and typically developing)

\section{Intragroup Comparisons}

A significant difference was found between the different subtests $\left(F_{(3,87)}=6.74, p=0.001, \mathrm{y}^{2}=0.18\right)$ in participants with high-functioning ASD. The post hoc tests revealed the mean scores of the Forward Memory (mean=8.17, $\mathrm{SD}=2.06$ ) considerably greater than the other subtests.

Also in TD participants, a significant difference was found between the different subtests $\left(F_{(3,87)}=21.49\right.$, $\left.\mathrm{p}=0.001, \mathrm{p}^{2}=0.42\right)$. The post hoc tests revealed the mean scores of the Associated Pairs (mean=8.17, SD=2.06) significantly lower than the other subtests.

The Correlations between WM performance and ASD prominent deficit in participants with high-functioning ASD

As shown in Table 2, the Pearson's correlation coefficient exhibited a significant positive correspondence between the Social interaction-standard score of the GARS-2 and the Forward Memory subtest $(\mathrm{r}=0.389 \mathrm{p}=0.034)$ (Fig. 2) and the Reverse Memory subtest $(r=0.387, p=0.035)$

Table 1. Characteristics of the high-functioning ASD participants

\begin{tabular}{|c|c|c|c|c|c|}
\hline \multirow[b]{2}{*}{ Items } & \multicolumn{2}{|c|}{ ASD $(\mathrm{N}=30)$} & \multicolumn{2}{|c|}{$\mathrm{TD}(\mathrm{N}=30)$} & \multirow{2}{*}{$\begin{array}{c}\mathrm{p} \\
\text { (ASD vs. TD) }\end{array}$} \\
\hline & Mean (SD) & Range & Mean (SD) & Range & \\
\hline Age (mounts) & $133.17(33.12)$ & $70-120$ & $134.43(32.06)$ & $91-128$ & 0.881 \\
\hline FSIQ (WISC-IV) & $85.63(12.51)$ & $83-184$ & $111.87(9.62)$ & $83-184$ & 0.001 \\
\hline GARS-2 Stereotyped Behaviors SS & $6.1(2.66)$ & $3-12$ & - & - & - \\
\hline GARS-2 Communication SS & $4.2(1.38)$ & $1-7$ & - & - & - \\
\hline GARS-2 Social interaction SS & $6.1(1.56)$ & $3-10$ & - & - & - \\
\hline GARS-2 Autism Index & $74.16(11.85)$ & $58-102$ & - & - & - \\
\hline
\end{tabular}

AARS-2 Autism Index

ASD: Autism Spectrum Disorder

TD: Typical Development

FSIQ: Full-Scale intelligence quotient

SES: Socioeconomic Status

WISC-IV: Wechsler Intelligence Scale for Children-Fourth Edition

GARS-2: Gilliam Autism Rating Scale-Second Edition

SS: Standard Score

Table 2. Pearson's correlations between the WM tasks of Leiter-R and ASD symptomatology

\begin{tabular}{|c|c|c|c|c|c|c|c|c|}
\hline \multirow[b]{2}{*}{ Scale } & \multicolumn{2}{|c|}{ Associated Pairs } & \multicolumn{2}{|c|}{ Forward Memory } & \multicolumn{2}{|c|}{ Reverse Memory } & \multicolumn{2}{|c|}{ Spatial Memory } \\
\hline & $\begin{array}{c}\text { Pearson } \\
\text { Correlation }\end{array}$ & $\mathrm{p}$ & $\begin{array}{c}\text { Pearson } \\
\text { Correlation }\end{array}$ & $\mathrm{p}$ & $\begin{array}{c}\text { Pearson } \\
\text { Correlation }\end{array}$ & $\mathrm{p}$ & $\begin{array}{c}\text { Pearson } \\
\text { Correlation }\end{array}$ & $\mathrm{p}$ \\
\hline GARS-2 Stereotyped Behaviors SS & 0.02 & 0.90 & 0.20 & 0.27 & 0.15 & 0.41 & 0.23 & 0.21 \\
\hline GARS-2 Communication SS & -0.14 & 0.43 & -0.04 & 0.80 & 0.01 & 0.93 & -0.23 & 0.20 \\
\hline GARS-2 Social interaction SS & 0.13 & 0.47 & $0.38 *$ & 0.03 & $0.38 *$ & 0.03 & 0.30 & 0.09 \\
\hline GARS-2 Autism Index & 0.01 & 0.94 & 0.23 & 0.21 & 0.21 & 0.26 & 0.16 & 0.39 \\
\hline
\end{tabular}




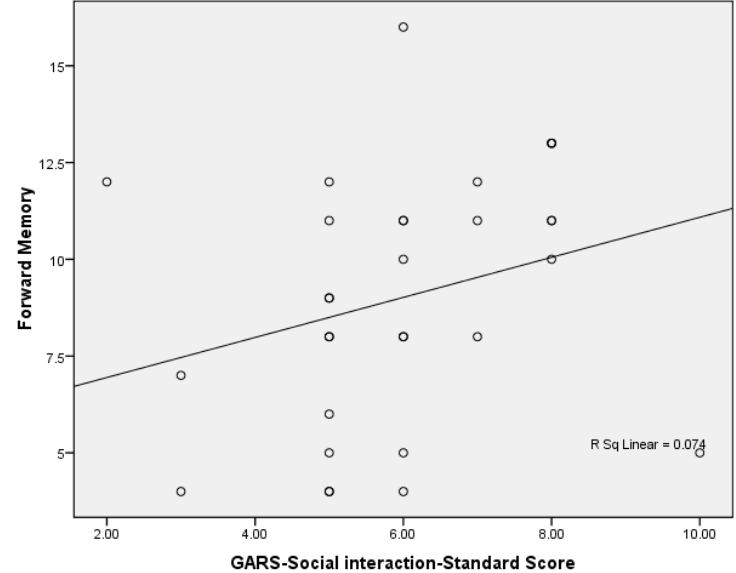

Fig. 2. Correlation of Forward Memory task and Gilliam Autism Rating Scale-Second Edition-Social interaction

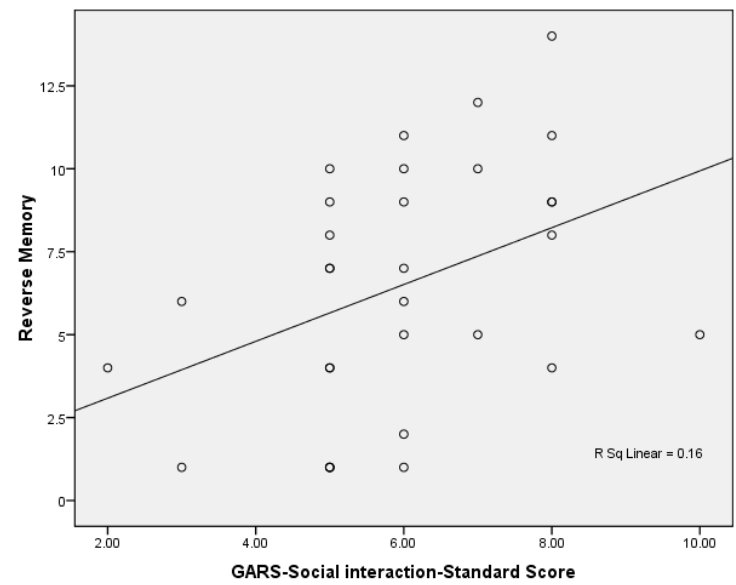

Fig. 3. Correlation of Reverse Memory task and Gilliam Autism Rating Scale-Second Edition-Social interaction

(Fig. 3).

\section{Discussion}

The aim of the current study was to compare nonverbal working memory capacity in persons with high functioning ASD and typically developing peers.

Working memory performances of the two groups were compared in 4 subtests: Associated pair subtest, Forward Memory subtest, Reverse Memory subtest and Spatial Memory subtest. Two groups were compared in two conditions: in the first condition, FSIQ wasn't controlled between two groups and in the second one FSIQ was controlled between two groups. Two groups had a significant difference in four subtests in the first condition, but there was a significant difference between the two groups only for Reverse Memory in the second condition.

The significant differences between the two groups in WM subtests showed general WM impairment in individuals with high functioning ASD. This finding is consistent with the results of most past studies in developed countries $(7,14)$. In neuropsychological studies, to justify the WM impairment in people with ASD, structural abnormality and hyperconnectivity in the brain need to be considered which is contributing to the cognitive function of ASD (32).

The results of the present study revealed that if the effect of FSIQ was controlled in the two groups, the Reverse Memory subtest could detect WM impairment. In order to interpret this finding the four WM subtests of the Leiter-r were compared. Association pair subtest measures the performance of long-term retrieval as a broad ability. Three remaining subtests measure visuospatial WM (33). Some studies reported that individuals with ASD had relatively intact long-term memory $(34,35)$, but behavioral $(7,14)$ and electrophysiological studies (36) indicate that persons with ASD had visuospatial memory deficits.

Among the three tasks used in this research for the evaluation of visuospatial WM, Reverse Memory task is more complex than Spatial Memory task (19) and need more cognitive load than Forward Memory task. Reverse Memory involves both brief holding of information and manipulating them, but Forward Memory task involves only holding the information (37). These findings were consistent with the results of the previous study that found children with ASD were significantly poorer performance than the FSIQ matched TD participants in the Backward Spatial Span but not in the Forward Spatial Span (38). Past studies support this notion that individuals with ASD have more challenges in performing more complex tasks $(14,32)$.

In participants with high-functioning ASD, better performance was found in the Forward Memory subtest, and a relative weakness was found in TD participants in the Associated Pairs subtest. As mentioned above, the Associated Pairs subtest explores long-term memory(33). It seems that in TD participants' cognitive load and complexity had no significant effect on visuospatial WM tasks and long-term memory task is more challenging than visuospatial WM tasks. However different profile was detected in participants with ASD.

Unpredictable results in the present study were a significant positive correlation between the GARS-2 subscale of Social interaction with the Forward Memory and Reverse Memory subtests of Leiter-R. In other words, the higher parental reported social problems were correlated with a score of the Forward Memory and Reverse Memory subtests. It should be noted that a small number of studies that examine the WM impairment in individuals with high-functioning ASD have reported the relationship between autism symptoms and working memory impairment (7). The results of some studies showed a negative correlation between ASD prominent deficit and WM performance $(11,39,40)$ but others did not find this relationship (41). Given that few studies have investigated this relationship, limited WM tasks have been used to examine this relationship. Given the impact of the format of the WM task on the performance of people with ASD so, it could be concluded that this relationship can also alternate based on the nature of different tasks. Of course, it should also be noted that in this sample, the relationship between autism prominent deficit and the WM subtests of the WISC-IV Integrated were also examined (unpublished study). These results also showed a significant positive correlation between the Social interaction subscale of the GARS-2 and the Digit Span Forward subtest. Also, the GARS-2 Stereotyped Behaviors subscale 
showed a significant positive correlation with the Visual Digit Span task. Although due to the limitations of the study, these results should be interpreted with caution. An important point in these findings is the value of examining it in future research.

\section{Limitations}

The results of this study should be reevaluated in larger sample size using different WM tasks in various communities to reach a definitive result. One limitation of this study was the difference between the two groups in terms of their FSIQ. Since the FSIQ of participants with ASD is mostly lower than their age-matched TD group, matching the FSIQ of the two groups was rather difficult (42). So, in the present study, statistical analysis was used to control the effect of FSIQ similar to previous studies conducted in this area and not able to match the two groups from the baseline for FSIQ (43-45). Try to raise some more points which needed to be addressed in the next studies.

\section{Implications of the findings for future research}

The results of the present study will have important implications for to understand the impact of high-functioning ASD performance on nonverbal WM profiles and to identify the patterns of relative strengths and weaknesses of WM in children with high-functioning ASD, and to guide intervention and educational programming.

\section{Conclusion}

The results obtained in this study exhibited that the Reverse Memory task could detect WM impairment in participants with high-functioning ASD compared to TD group in the recruited samples if the effect of FSIQ were controlled. This means that the design of WM tasks may regulate whether or not WM performance is impaired. Participants with high-functioning ASD may have more problems when task complexity and cognitive load increased. Also, the profile of WM subtests of Leiter-R in participants with high-functioning ASD and TD group were different. The point to be considered in this research is that there is a significant positive correlation between two WM subtests of Leiter-R and subscale of Social interaction GARS-2. Further research with more sample sizes can reevaluate the results of the present study.

\section{Acknowledgments}

This article was one part of the first author's Ph.D. dissertation in speech and language pathology and supported by Iran University of Medical Sciences. The authors would like to state gratitude to all participants involved in the study.

\section{Conflict of Interests}

The authors declare that they have no competing interests.

\section{References}

1. American Psychiatric Association. Diagnostic and statistical manual of mental disorders (DSM-5®). American Psychiatric Pub; 2013 May 22 .
2. Sanders JL. Qualitative or quantitative differences between Asperger's disorder and autism? Historical considerations. J Autism Dev Disord. 2009 Nov 1;39(11): 1560.

3. Samadi SA, McConkey R. Screening for autism in Iranian preschoolers: Contrasting M-CHAT and a scale developed in Iran. J Autism Dev Disord. 2015 Sep 1;45(9):2908-16.

4. Fombonne E. Epidemiology of pervasive developmental disorders. Pediatr. Res. 2009 Jun;65(6):591.

5. Rappaport SR, Kanter C, Anast K. Special Considerations in Child Custody Evaluations for Children with High-Functioning Autism Spectrum Disorder. Fam Court Rev. 2016 Jan;54(1):81-95.

6. Cumine V, Dunlop J, Stevenson G. Autism in the early years: A practical guide. Routledge; 2009 Dec 4.

7. Wang Y, Zhang YB, Liu LL, Cui JF, Wang J, Shum DH, et al. A metaanalysis of working memory impairments in autism spectrum disorders. Neuropsychol Rev. 2017 Mar 1;27(1):46-61.

8. Baddeley A. Working memory. Science. 1992 Jan 31;255(5044):556-

9. Dehn MJ. Working memory and academic learning: Assessment and intervention. John Wiley \& Sons; 2011 Jan 4.

10. Gilotty L, Kenworthy L, Sirian L, Black DO, Wagner AE. Adaptive skills and executive function in autism spectrum disorders. Child Neuropsychol. 2002 Dec 1;8(4):241-8.

11. Lopez BR, Lincoln AJ, Ozonoff S, Lai Z. Examining the relationship between executive functions and restricted, repetitive symptoms of autistic disorder. J Autism Dev Disord. 2005 Aug 1;35(4):445-60.

12. Bodner KE, Beversdorf DQ, Saklayen SS, Christ SE. Noradrenergic moderation of working memory impairments in adults with autism spectrum disorder. J Int Neuropsychol Soc. 2012 May;18(3):556-64.

13. Morsanyi K, Holyoak KJ. Analogical reasoning ability in autistic and typically developing children. Dev Sci. 2010 Jul;13(4):578-87.

14. Kercood S, Grskovic JA, Banda D, Begeske J. Working memory and autism: A review of literature. Res Autism Spectr Disord. 2014 Oct 1;8(10):1316-32.

15. Roid GH, Miller LJ. Leiter international performance scale-revised: Examiners manual: Stoelting Wood Dale.

16. Michalec D. Leiter International Performance Scale: Revised. In Encyclopedia of Child Behavior and Development 2011 (pp. 882-882). Springer, Boston, MA.

17. Tsatsanis KD, Dartnall N, Cicchetti D, Sparrow SS, Klin A, Volkmar FR. Concurrent validity and classification accuracy of the Leiter and Leiter-R in low-functioning children with autism. J Autism Dev Disord. 2003 Feb 1;33(1):23-30

18. Kuschner ES, Bennetto L, Yost K. Patterns of nonverbal cognitive functioning in young children with autism spectrum disorders. J Autism Dev Disord. 2007 May 1;37(5):795-807.

19. Baker S, Hooper S, Skinner M, Hatton D, Schaaf J, Ornstein P, et al. Working memory subsystems and task complexity in young boys with Fragile X syndrome. J Ment Defic Res. 2011 Jan;55(1):19-29.

20. Hooper SR, Hatton DD, Baranek GT, Roberts JP, Bailey Jr DB. Nonverbal assessment of IQ, attention, and memory abilities in children with fragile-X syndrome using the Leiter-R. J Psychoeduc Assess. 2000 Sep;18(3):255-67.

21. Hedden T, Ketay S, Aron A, Markus HR, Gabrieli JD. Cultural influences on neural substrates of attentional control. Psychol Sci. 2008 Jan;19(1):12-7.

22. Montgomery JM, Newton B, Smith C. Test Review: Gilliam, J.(2006). GARS-2: Gilliam Autism Rating-Scale-Second Edition. Austin, TX: PRO-ED. J Psychoeduc Assess. 2008 Dec;26(4):395-401

23. Wechsler D. Wechsler intelligence scale for children-Fourth Edition (WISC-IV). San Antonio, TX: The Psychological Corporation. 2003.

24. García-Villamisar D, Sala SD. Dual-task performance in adults with autism. Cog Neuropsychiatry. 2002;7(1):63-74.

25. Sadeghi A, Rabiee M, Abedi MR. Validation and reliability of the Wechsler intelligence scale for children-IV. Develop Psychol. 2011;7(28):47-55. (Persian)

26. Samadi SA, McConkey R. The utility of the Gilliam autism rating scale for identifying Iranian children with autism. Disabil Rehabil. 2014 Mar 1;36(6):452-6.

27. Goodman R. The Strengths and Difficulties Questionnaire: a research note. J Child Psychol Psychiat. 1997 Jul;38(5):581-6.

28. Shahrivar Z, Tehrani-Doost M, Pakbaz B, Rezaie A, Ahmadi F. Normative data and psychometric properties of the parent and teacher versions of the strengths and difficulties questionnaire (SDQ) in an Iranian community sample. J Res Med Sci. 2009 Mar;14(2):69. 
29. Ghanizadeh A, Izadpanah A, Abdollahi G. Scale validation of the strengths and difficulties questionnaire in Iranian children. Iran J Psychiat. 2007;2(2):65-71.

30. Kazemipour Sh. A pattern to determine the social economic status and measure the social move-based on a case study in Tehran. Soc Sci Let. 1999 Dec;14(14). (Persian)

31. Willcutt EG, Pennington BF, Boada R, Ogline JS, Tunick RA, Chhabildas NA, et al. A comparison of the cognitive deficits in reading disability and attention-deficit/hyperactivity disorder. J Abnorm Psychol. $2001 \mathrm{Feb} ; 110(1): 157$.

32. Barendse EM, Hendriks MP, Jansen JF, Backes WH, Hofman PA, Thoonen G, et al. Working memory deficits in high-functioning adolescents with autism spectrum disorders: neuropsychological and neuroimaging correlates. J Neurodev Disord. 2013 Dec;5(1):14.

33. McCallum RS, editor. Handbook of nonverbal assessment. New York, NY: Kluwer Academic/Plenum Publishers; 2003 May 1.

34. Bennetto L, Pennington BF, Rogers SJ. Intact and impaired memory functions in autism. Child Dev. 1996 Aug;67(4):1816-35.

35. Renner P, Klinger LG, Klinger MR. Implicit and explicit memory in autism: Is autism an amnesic disorder? J Autism Dev Disord. 2000 Feb $1 ; 30(1): 3-14$

36. Gruber O, von Cramon DY. The functional neuroanatomy of human working memory revisited: Evidence from 3-T fMRI studies using classical domain-specific interference tasks. Neuroimage. $2003 \mathrm{Jul}$ 1;19(3):797-809.

37. Flanagan DP, Ortiz SO, Alfonso VC. Essentials of cross-battery assessment. John Wiley \& Sons; 2013 Mar 6.

38. Joseph RM, McGrath LM, Tager-Flusberg H. Executive dysfunction and its relation to language ability in verbal school-age children with autism. Dev Neuropsychol. 2005 Jun 1;27(3):361-78.

39. Landa RJ, Goldberg MC. Language, social, and executive functions in high functioning autism: A continuum of performance. J Autism Dev Disord. 2005 Oct 1;35(5):557.

40. Sachse M, Schlitt S, Hainz D, Ciaramidaro A, Schirman S, Walter H, et al. Executive and visuo-motor function in adolescents and adults with autism spectrum disorder. J Autism Dev Disord. 2013 May 1;43(5):1222-35.

41. Steele SD, Minshew NJ, Luna B, Sweeney JA. Spatial working memory deficits in autism. J Autism Dev Disord. 2007 Apr 1;37(4):605-12.

42. Salmanian M, Tehrani-Doost M, Ghanbari-Motlagh M, Shahrivar Z. Visual memory of meaningless shapes in children and adolescents with autism spectrum disorders. Iran J Psychiat. 2012;7(3):104.

43. Goldberg MC, Mostofsky SH, Cutting LE, Mahone EM, Astor BC, Denckla MB, et al. Subtle executive impairment in children with autism and children with ADHD. J Autism Dev Disord. 2005 Jun 1;35(3):279-93.

44. Geurts HM, Verté S, Oosterlaan J, Roeyers H, Sergeant JA. How specific are executive functioning deficits in attention deficit hyperactivity disorder and autism? J Child Psychol Psychiat. 2004 May;45(4):83654.

45. Verte S, Geurts HM, Roeyers H, Oosterlaan J, Sergeant JA. Executive functioning in children with autism and Tourette syndrome. Dev. Psychopathol. 2005 Jun;17(2):415-45. 